Int. J. Morphol.,

28(1):13-18, 2010

\title{
¿Existe Modelamiento Profesional de los Estilos de Aprendizaje?
}

\author{
Professional Modeling of Learning Styles does Exist?
}

\author{
"Iván Suazo Galdames; **Andrea Precht Gandarilla; "Mario Cantín López; \\ ****Daniela Zavando Matamala \& *Catherine Sandoval Marchant
}

\begin{abstract}
SUAZO, G. I.; PRETCH, G. A.; CANTÍN, L. M.; ZAVANDO, M. D. \& SANDOVAL, M. C. ¿Existe modelamiento profesional de los estilos de aprendizaje? Int. J. Morphol., 28(1):13-18, 2010.

RESUMEN: La determinación de los estilos de aprendizaje (EA) ha sido una preocupación pedagógica permanente, indicando la forma en que cada estudiante estudia y aprende, permitiendo modificar el diseño y la implementación de los procesos de aprendizaje, haciéndolos más eficaces en la formación de un profesional de la salud. En el presente estudio se analizaron las diferencias de los EA en un grupo de alumnos de Kinesiología, medidos en el primer y último años de su Carrera profesional. Se diseñó un estudio exploratorio descriptivo longitudinal cuantitativo mediante la aplicación del Cuestionario de EA Honey-Alonso (CHAEA) en estudiantes de Kinesiología, Universidad de Talca. Un primer análisis de los estilos de aprendizaje fue realizado en 48 estudiantes, el primer semestre de su Carrera, el año 2003 y luego un segundo análisis en 38 de estos alumnos el último semestre de su Carrera el 2007. La prueba t para muestras relacionadas fue utilizada para establecer su significancia. Los alumnos presentaron todos los EA. El de mayor predominio fue el estilo reflexivo, luego el teórico, el pragmático y finalmente el activo. Las preferencias de estilos de aprendizaje reflexivo, pragmático y activo no tuvieron diferencias significativas entre los años 2003-2007. Sólo el EA teórico aumentó sus preferencias el año 2007. Nuestra investigación recalca la importancia de determinar las formas como los estudiantes estudian y aprenden durante los primeros años de su carrera. Esta información puede ser utilizada para diseñar estrategias metodológicas que favorezcan la adquisición de mejores competencias profesionales.
\end{abstract}

PALABRAS CLAVE: Estilo de aprendizaje; Modelamiento profesional; Cuestionario CHAEA.

\section{INTRODUCCIÓN}

En las últimas décadas, el análisis de la forma en que los estudiantes estudian y aprenden ha sido una preocupación pedagógica permanente. Prueba de ello son la gran cantidad de instrumentos desarrollados para determinar los estilos de aprendizaje (EA) preferidos por los estudiantes (Gregorc \& Ward, 1977; Gregorc, 1979, 1985; Dunn et al., 1982; Dunn \& Dunn, 1989; Kolb, 1984; Felder \& Silverman, 1988; Entwistle \& Tait, 1995; Fleming, 2001; Duff, 2004), la mayoría de los cuales se basa en la idea que los estilos de aprendizaje presentan una cierta estabilidad, pero que en el desarrollo de las competencias profesionales estos se van haciendo cada vez mas complejos, o bien. estos estilos de aprendizaje pueden ser modelados durante el periodo de estudios universitarios (Canaleja et al., 2005; Villalobos et al., 2009).
El concepto de estilo de aprendizaje tiene múltiples definiciones pero todas coinciden en que se trata de cómo el cerebro procesa la información y cómo es influida por las percepciones de cada individuo (Messick, 1969; Witkin, 1975).

De acuerdo a Alonso et al. (1997) los estilos de aprendizaje son los rasgos cognitivos, afectivos y fisiológicos, que sirven como indicadores relativamente estables, de cómo los discentes perciben, interaccionan y responden a sus ambientes de aprendizaje.

Para Honey (1983) lo ideal sería que todo el mundo fuera capaz de experimentar, reflexionar, elaborar hipótesis y aplicar a partir de la experiencia, de manera equivalente.

* Departamento de Morfología, Facultad de Ciencias de la Salud, Universidad de Talca, Chile.

** Directora Centro de Innovación y Calidad de la Docencia (CICAD), Universidad de Talca, Chile.

****Universidad Autónoma de Chile, Sede Talca, Chile. 
Pero lo cierto es que los individuos raramente poseen todas estas cualidades de manera uniforme. Los estilos de aprendizaje, entonces, serán algo así como la interiorización por parte de cada sujeto, de una etapa determinada del ciclo de aprendizaje.

Las implicaciones de entender los estilos de aprendizaje como elementos dinámicos son significativas. Si una institución educativa está consciente que sus estudiantes tienen distintas formas de abordar la experiencia, deberá incorporar este conocimiento al diseño y a la implementación de los procesos de aprendizaje, con el fin de que éstos sean más eficaces (Maldonado-Rojas, 2008).

En el transcurso de la formación de un profesional de la salud, el estudiante debe interactuar con profesores de distintas áreas, los cuales a su vez poseen distintos estilos de enseñanza. El concepto estilos de enseñanza es un constructo que busca describir las interacciones que existen entre el profesor y el estudiante (Fischer \& Fischer, 1969); esta definición fue ampliada por Smith (1997) quien definió operacionalmente el concepto estilo de enseñanza de calidad, como aquellas acciones, interacciones y comunicaciones del docente con sus estudiantes, que se asocian a progresos cognitivos y/o afectivos en ellos.

Fisher \& Fisher definen el estilo de enseñar "como un modo habitual de acercarse a los discentes con varios métodos de enseñanza". De esta definición se desprende claramente la relación entre los conceptos antes mencionados, estilos de aprendizaje y estilos de enseñanza, a las cuales podríamos agregar un tercer concepto relacionado, las estrategias o métodos de enseñanza. El docente ante el proceso de enseñanza aprendizaje puede, según su estilo de enseñanza, aplicar una cierta estrategia de enseñanza, o bien desde otra perspectiva, según los estilos de aprendizaje de los estudiantes el docente tendrá que buscar las estrategias de enseñanza adecuadas y modelarlas a su propio estilo.

En el presente estudio se analizan las diferencias en los estilos de aprendizaje, en un grupo de alumnos de Kinesiología, medidos en el primer y último años de su Carrera Profesional.

\section{MATERIAL Y MÉTODO}

Se diseñó un estudio exploratorio descriptivo longitudinal cuantitativo, que utilizó datos empíricos obtenidos mediante la aplicación a los estudiantes del Cuestionario de estilos de aprendizaje de Honey-Alonso (CHAEA).
En este estudio participaron estudiantes de la carrera de Kinesiología de la Universidad de Talca, Chile.

Un primer análisis de los estilos de aprendizaje se realizó en 48 estudiantes (90,5\% del total matriculado) que se encontraban cursando el primer semestre de su Carrera el año 2003 y un segundo análisis se realizó en un grupo de 38 alumnos que se encontraban en el último semestre de su Carrera el año 2007 y que pertenecían al grupo anteriormente mencionado.

Las comparaciones entre los resultados se realizaron, por tanto, en los 38 sujetos a los cuales se les aplicó el cuestionario tanto en el año 2003 como en el 2007.

Instrumento. El Cuestionario de estilos de aprendizaje de Honey-Alonso (CHAEA) es el instrumento que se utilizó para medir e identificar los estilos de aprendizaje que predominaban en el grupo de estudiantes de Kinesiología de la Universidad de Talca.

El cuestionario consta de 80 ítems breves y se estructuran en cuatro grupos o secciones de 20 ítems correspondientes a los cuatro estilos de aprendizaje (activo, reflexivo, teórico y pragmático). Todos los ítems están distribuidos aleatoriamente formando un solo conjunto.

La validación del cuestionario había sido reportada previamente por Suazo (2007), para el análisis de la confiabilidad se utilizó el alfa de Cronbach. Los datos fueron procesados mediante el uso del programa SPSS 11.5 para Windows, con el que se obtuvieron los estadísticos descriptivos para los estilos de aprendizaje. La prueba t para muestras relacionadas con un intervalo de confianza del 95\% se utilizó para establecer la significancia de las relaciones.

\section{RESULTADOS}

En el año 2003, el Cuestionario de Estilos de Aprendizaje de Honey y Alonso et al. fue aplicado a un grupo de 48 estudiantes, los que en la segunda aplicación, el año 2007, disminuyeron a 38 , por lo que los resultados aquí analizados corresponden a aquellos estudiantes que respondieron el cuestionario el año 2003 cuando cursaban el primer año y el año 2007 cuando cursaban el quinto año de la carrera de Kinesiología. La muestra final, por tanto, quedó conformada por 38 alumnos.

Los resultados que se obtuvieron del CHAEA muestran que los alumnos pertenecientes a la muestra presentan todos los estilos de aprendizaje, pero en distinta medida, 
existiendo casos en que no hay gran diferencia entre un estilo y otro, por lo cual, en el presente estudio se ha decidido no encasillar al alumno en el estilo con mayor puntuación, sino que, se analiza la media obtenida de los alumnos en cada uno de los estilos de aprendizaje.

Análisis de los estilos de aprendizaje. Todos los estudiantes manifiestan uso o preferencias de distintos estilos de aprendizaje, sin embargo, el de mayor predominio es el estilo reflexivo $(\mathrm{R})$, luego el teórico $(\mathrm{T})$, el pragmático $(\mathrm{P})$ $\mathrm{y}$ finalmente el estilo activo (A), aunque con diferencias de los valores promedio de los estilos de aprendizaje tuvieron un comportamiento similar en las dos oportunidades en que se aplicó el cuestionario. Los estadísticos descriptivos de los estilos de aprendizaje para los años 2003 y 2007 se epresentan en la Tabla I.

Para analizar si la preferencia entre los estilos de aprendizaje difiere entre ellos de manera significativa, se aplicó la prueba de hipótesis no paramétrica de Friedman $(\mathrm{p}=0,01 \%)$. Mediante esta prueba se analiza si la preferencia entre los estilos de aprendizaje difiere entre ellos de manera significativa $(\mathrm{p}=0,01 \%)$, arrojando un valor de chi cuadrado $=37,732$ (2007) y 48,690 (2003), por lo tanto, aunque todos los estilos tienen una preferencia moderada, existe evidencia suficiente para pensar que al menos dos estilos de aprendizaje (por ejemplo, el teórico y el reflexivo) difieren significativamente en cuanto a su preferencia.

Para analizar si las preferencias por los estilos de aprendizaje activo (A), reflexivo (R), teórico (T) y pragmático (P) analizados el año 2003 eran significativamente distintas a los valores obtenidos el año 2007, aplicamos la prueba t para muestras relacionadas, no encontrándose diferencias significativas en los estilos reflexivo, pragmático y activo. El estilo de aprendizaje teórico aumentó sus preferencias, el año 2007 y esta diferencia resultó significativa. Los estadísticos para la prueba t se muestran en la Tabla II.

Tabla I. Estadísticos descriptivos de los estilos de aprendizaje para los años 2003 y 2007. A= Activo, $\mathrm{R}=$ Reflexivo, $\mathrm{T}=$ Teórico, $\mathrm{P}=$ Pragmático.

\begin{tabular}{lcrccc}
\hline & n & Mínimo & Máximo & Media & Desv. Típ. \\
\hline A2007 & 38 & 6,00 & 16,00 & 10,7632 & 2,99941 \\
R2007 & 38 & 10,00 & 20,00 & 15,5789 & 2,93747 \\
T2007 & 38 & 6,00 & 19,00 & 14,5789 & 2,99217 \\
P2007 & 38 & 7,00 & 17,00 & 12,9737 & 2,44382 \\
A2003 & 38 & 4,00 & 17,00 & 10,6316 & 2,97213 \\
R2003 & 38 & 11,00 & 20,00 & 15,6579 & 2,32816 \\
T2003 & 38 & 9,00 & 17,00 & 13,1842 & 1,90100 \\
P2003 & 38 & 5,00 & 19,00 & 12,2895 & 2,62944 \\
\hline
\end{tabular}

Tabla II. Estadísticos para la prueba t de preferencias según estilos de aprendizaje entre los años 2003 y 2007. A= Activo, R= Reflexivo, $\mathrm{T}=$ Teórico, $\mathrm{P}=$ Pragmático.

\begin{tabular}{llllcl}
\hline & & Media & n & Desviación Típ. & 0,815 \\
\hline Par 1 & A2007 & 10,7632 & 38 & 2,99941 & 2,97213 \\
Par 2 & A2003 & 10,6316 & 38 & 2,93747 & 0,856 \\
& R2007 & 15,5789 & 38 & 2,32816 & 0,002 \\
Par 3 & R2003 & 15,6579 & 38 & 2,99217 & 1,90100 \\
& T2007 & 14,5789 & 38 & 2,44382 & 0,135 \\
& T2003 & 13,1842 & 38 & 2,62944 & \\
\hline
\end{tabular}




\section{DISCUSIÓN}

Los estilos de aprendizaje son indicadores de cómo el alumno se aproxima al contenido con el fin de lograr el aprendizaje. En la formación de profesionales de la salud se utilizan diversas estrategias para lograr que los estudiantes sean profesionales competentes, algunas de las cuales ponen su énfasis en los aspectos teóricos, otras en lo prácticos, otras en la resolución de problemas, etc. Diversos autores (Inzunza \& Bravo, 1999; Guiraldes et al., 1995; Inzunza et al., 1993) se han referido a la utilización de distintas estrategias metodológicas para la enseñanza de la Anatomía Macroscópica, determinando que muchas eran eficientes como apoyo para la comprensión de los contenidos prácticos, conclusión similar a la que llegaron Rojas et al. (1994) en el campo de la Histología, atribuyendo a las características propias de los estudiantes y de cómo éstos abordan la experiencia, lo que cobra la mayor importancia a la hora de obtener aprendizajes significativos.

Variados autores se han referido a estrategias para la formación de profesionales de la salud y la relación de éstas con los estilos cognitivos y el desarrollo de competencias clínicas (Fox, 1984; Ghali et al., 1998; Taylor \& Buterakos, 1998; Green, 1999; Curry, 1999, 2000; Sackett et al., 2000; Souza et al., 2004; Escanero et al., 2009). En este estudio se analizaron las preferencias por los estilos de aprendizaje que mostraron los estudiantes de la Carrera de Kinesiología, al inicio de su Carrera y luego al final de ella.

El análisis de la distribución de los estilos de aprendizaje permite aseverar que no existe un estilo que predomine por sobre los otros, aún cuando existió un mayor número de preferencias de los estudiantes por el estilo reflexivo, esta diferencia no resultó estadísticamente significativa debido a que un estudiante podía manifestar preferencia por un estilo de aprendizaje particular, pero asignarle valores importantes a los otros estilos.

Cuando en el presente estudio se analizaron las correlaciones existentes en los estilos de aprendizaje entre sí, no se observaron diferencias significativas en la preferencia de los estilos de aprendizaje reflexivo, pragmático y activo, atribuibles al modelamiento profesional, o a los 5 años de formación profesional, hecho que puede considerarse como un antecedente de la estabilidad de los estilos de aprendizaje a través del tiempo, lo que coincide con diversos autores que se han referido al carácter estable de los estilos de aprendizaje (Honey; Cornwell \& Manfredo, 1994; Riding, 1994; Riding \& Rayner, 1998) como indicadores de cómo los individuos se relacionan con sus escenarios de aprendizaje. En ese mismo sentido, Martín-García no encontró diferen- cias significativas en los estilos de aprendizajes atribuibles al paso de los años. En contraste con estas opiniones, otros autores consideran que los estilos de aprendizaje son modelados durante el proceso de adquisición de competencias profesionales y el asentamiento de las personas en estilos preferidos o de mayor dominancia se debe a razones de comodidad y no le permiten desarrollar otras destrezas de aprendizaje propias de los otros estilos (Fox), mientras que Dorsey \& Pierson (1984) asocian los estilos de aprendizaje con la edad, ellos indican que los jóvenes tienden a tener un estilo más activo en sus enfoques de aprendizaje, mientras que los mayores tienden a ser más reflexivos y abstractos.

Resulta significativo que los estilos de aprendizaje preferidos por estudiantes de Kinesiología al inicio de su Carrera fueron los estilos reflexivo y teórico, y que luego de una formación, que en Kinesiología pone énfasis en la práctica (Souza et al.), favorable para el desarrollo de los estilos pragmático y activo, no se hayan modificado las preferencias por el estilo reflexivo y hayan aumentado para el estilo teórico, resultados muy similares a los obtenidos por Borracci et al. (2008) en alumnos universitarios y médicos residentes. Por estas razones, el perfil de egreso de los estudiantes, incluye características que no pueden ser atribuidas al modelamiento profesional y que, probablemente, implicará distintos mecanismos de abordaje de los problemas en el ejercicio profesional y en su formación continua.

Los datos que se presentan en esta investigación recalcan la importancia de determinar las formas cómo los estudiantes estudian y aprenden durante los primeros años de su Carrera, ya que esta información puede ser utilizada para diseñar estrategias metodológicas que favorezcan la adquisición de competencias profesionales.

SUAZO, G. I.; PRETCH, G. A.; CANTÍN, L. M.; ZAVANDO, M. D. \& SANDOVAL, M. C. Professional modeling of learning styles does exist? Int. J. Morphol., 28(1):13-18, 2010.

SUMMARY: The identification of learning styles has been a permanent teacher preoccupation, showing how each student learns and studies, allowing to modify the design and implementation of learning processes, making them more effective in training a health professional. The present study analyzed the differences of the learning styles in a group of students in Kinesiology measured in the first and last years of his career. We designed a descriptive exploratory longitudinal quantitative study by applying the questionnaire learning styles Honey-Alonso (CHAEA) on Kinesiology students, Universidad de Talca. A first analysis of learning styles was conducted on 48 students the first 
half of his career in 2003 and then a second analysis in 38 of these students in the latter half of his career in 2007. $t$ test for related samples was used to establish its significance. The students had all learning styles. The prevalence was higher in reflexive style, then theoretical, pragmatic and finally active styles. Preferences for reflexive, pragmatic and active learning styles had no significant differences between the years 2003-2007. Only the theoretical style increased their preferences in 2007. Our research highlights the importance of identifying the ways students study and learn during the early years of his career, this information may be used to develop methodological strategies that promote the acquisition of better skills.

KEY WORDS: Learning style; Professional modelling; CHAEA questionnaire.

\section{REFERENCIAS BIBLIOGRÁFICAS}

Alonso, C.; Gallego, D. \& Honey, P. Los estilos de aprendizaje. Procedimientos de diagnóstico y mejora. Bilbao, Ediciones Mensajero, 1997.

Borracci, R.; Guthman, G.; Rubio, M. \& Arribalzaga, E. Estilos de aprendizaje en estudiantes universitarios y médicos residentes. Educ. Med. 11:229-38, 2008.

Canaleja, M.; Martínez, M.; Pineda, M.; Vera, M.; Sotop, M. \& Martín, A. Estilos de aprendizaje en los estudiantes de Enfermería. Educ. Med., 8:33-40, 2005.

Cornwell, J. M. \& Manfredo, P.A. Kolb's learning style theory revisited. Educ. Psychol. Meas., 54:317-27, 1994.

Curry, L. Cognitive and learning styles in medical education. Acad. Med., 74:409-13, 1999.

Curry, L. Review of learning style, studying approach, and instructional preference research in medical education. In Riding, R. J. \& Rayner, S. G. (Eds.). International perspectives on individual differences. Stamford, Ablex, 2000.

Dorsey, O. L. \& Pierson, M. S. A descriptive study of adult learning styles in a nontraditional education program. Lifelong Learning, 7:8-11, 1984.

Duff, A. The revised approaches to studying inventory (RASI) and its use in management education. Active Learning in Higher Education, 5(1):56-72, 2004.

Dunn, R. \& Dunn, K. Learning style inventory. Lawrence, Price Systems, 1989.
Dunn, R.; Dunn, K. \& Price, G. E. Productivity environmental preference survey. Lawrence, Price Systems, 1982.

Entwistle, N. J. \& Tait, H. The revised approaches to studying inventory. Centre for Research on Learning and Instruction. Edinburgh, University of Edinburgh, 1995.

Escanero, J.; González, C.; Ezquerra, L. \& Borque, L. Diseño e implementación de una práctica de metacognición en la asignatura de Fisiología Humana (Facultad de Medicina). Educ. Med., 12:47-53, 2009.

Felder, R. M. \& Silverman, L. K. Learning styles and teaching styles in engineering education. Engr. Education, 78(7):674-81, 1988.

Fischer, B. B. \& Fischer, L. Toward individualized learning. Elem. Sch. J., 69:298-303, 1969.

Fleming, N. D. Teaching and learning styles: VARK strategies. Christchurch, Fleming, 2001.

Fox, R. D. Learning styles and instructional preferences in continuing education for health professionals: a validity study of the LSI. Adult. Educ. Q., 35:72-85, 1984.

Ghali, W. A.; Lesky, L. G. \& Hershman, W. Y. The missing curriculum. Acad. Med., 73:734-6, 1998.

Gregorc, A. F. Inside styles: Beyond the basics. Maynard, Gabriel Systems, 1985.

Gregorc, A. F. Learning/teaching styles: Their nature and effects. In Keefe, J. W. (Ed.). Student learning styles: Diagnosing and prescribing programs. Reston, National Association of Secondary School Principals, 1979. pp.19-26.

Gregorc, A. F. \& Ward, H. B. Implications for learning and teaching: A new definition for individual. NASSP Bulletin, 61(406):20-6, 1977.

Green, M. L. Graduate medical education training in Clinical Epidemiology, critical appraisal, and evidence-based medicine: A critical review of curricula. Acad. Med., 74:68694, 1999.

Guiraldes, H.; Oddó, H.; Ortega, X. \& Oyarzo, M. Métodos computacionales y gráficos de apoyo al aprendizaje de la Anatomía Humana. Rev. Chil. Anat., 13:67-71, 1995.

Honey, P. Learning Styles-Their relevance to training courses to trainer officer, 1983. Disponible en: http:// www.peterhoney.co.uk/Article/55. 
Inzunza, O. \& Bravo, H. Impacto de dos programas computacionales de Anatomía Humana en el rendimiento del conocimiento práctico de los alumnos. Rev. Chil. Anat., 17:205-9, 1999.

Inzunza, O.; Bravo, H.; Garcia-Huidobro, C.; Contreras, P.; Riquelme, S. \& Valenzuela, A. Software docente para la enseñanza en Anatomía. Rev. Chil. Anat., 11:81, 1993.

Kolb, D. A. Experiential learning. Experience as the source of learning and development. New Jersey, Prentice-Hall, 1984.

Maldonado-Rojas, M. Valoración de la docencia en un Módulo de Salud Pública basado en competencias. Educ. Med., 11:219-28, 2008.

Martín-García, A. V. Estilos de aprendizaje en la vejez. Un estudio a la luz de la teoría del aprendizaje experiencial. Rev. Esp. Geriatr. Gerontol., 38:258-65, 2003.

Messick, S. The criterion problem in the evaluation of instruction. New Jersey, Educational Testing Services, 1969.

Riding, R. J. Personal style awareness and personal development. Birmingham, Learning and Training Technology, 1994.

Riding, R. \& Rayner, S. Cognitive styles and learning strategies. London, David Fulton Publishers, 1998.

Rojas, M.; Montiel, E.; Montiel, J.; Ondarza, A. \& Rodríguez, H. Estudio comparado entre métodos de enseñanza tradicional y computacional en Histología. Rev. Chil. Anat., 17:81-5, 1999.

Sackett, D. L.; Straus, S. E.; Richardson, W. S.; Rosenberg, $W$. \& Haynes, R. B. Evidence-based medicine: How to practice and teach EBM. New York, Churchill Livingston, 2000.

Smith, J. Sweating to learn. The National Teaching and Learning Forum, 6(3):9-11, 1997.

Souza, R. B.; Carvalho, R. M.; Capelli, A. M. G.; Ribeiro, M. C.; Vandresen Filho, S.; Simionato, C.; Silva, L. H.; Oda, D. S. \& Lavado, E. L. Eficacia del entrenamiento teórico-práctico en Anatomía Palpatoria empleada a los estudiantes de la Carrera de Fisioterapia. Int. J. Morphol., 22:195-200, 2004.
Suazo, G. I. C. Estilos de aprendizaje y su correlación con el rendimiento académico en Anatomía Humana Normal. Int. J. Morphol., 25:367-73, 2007.

Taylor, D. \& Buterakos, J. Evidence-based medicine: Not as simple at it. Acad. Med., 73:1221-2, 1998.

Villalobos, E.; Guerrero, M.; Pérez, R.; Avendaño, A.; Ceballos, A.; Ortiz, J. \& Parra, C. Estilos de aprendizaje y metodologías de enseñanza en estudiantes de Obstetricia. Educ. Med., 12:43-6, 2009.

Witkin, H. A. Some implications of research on cognitive style for problem of education. In Whitehead, J. M. (Ed.). Personality and Learning. London, Hodder and Stoughton, 1975.

Dirección para correspondencia:

Prof. Dr. Iván Suazo Galdames

Departamento de Morfología

Facultad de Ciencias de la Salud.

Universidad de Talca

Avenida Lircay s/n oficina N¹04

CHILE

Telefono: 56-71-201576

E-mail: isuazo@utalca.cl

Recibido: 12-01-2010

Aceptado:22-02-2010 